Original paper

\title{
End-to-end dosimetric audit: A novel procedure developed for Irish HDR brachytherapy centres
}

\author{
Sarah Bassi $^{\mathrm{a}, *}$, Leanne Berrigan ${ }^{\mathrm{b}}$, Anysja Zuchora ${ }^{\mathrm{c}}$, Louise Fahy ${ }^{\mathrm{b}}$, Margaret Moore ${ }^{\mathrm{b}}$ \\ ${ }^{\text {a }}$ Azienda Sanitaria Universitaria Friuli Centrale, Udine, Italy \\ ${ }^{\mathrm{b}}$ Galway University Hospitals, Medical Physics and Bioengineering, Galway, Ireland \\ ${ }^{\mathrm{c}}$ Health Service Executive, Department of Medical Physics and Bioeengineering, Galway, Ireland
}

\section{A R T I C L E I N F O}

\section{Keywords:}

Dosimetric audit

HDR brachytherapy

3D printable materials

Ir-192 dosimetry

TLD

GafChromic films

Farmer chamber

\begin{abstract}
A B S T R A C T
Purpose: A dosimetric audit of Ir-192 high dose rate (HDR) brachytherapy remote after-loading units was carried out in 2019. All six brachytherapy departments on the island of Ireland participated in an end-to-end test and in a review of local HDR dosimetry procedures.

Materials and methods: A 3D-printed customised phantom was created to position the following detectors at known distances from the HDR source: a Farmer ionization chamber, GafChromic film and thermoluminescent dosimeters (TLDs). Dedicated HDR applicator needles were used to position an Ir-192 source at $2 \mathrm{~cm}$ distance from these detectors. The end-to-end dosimetry audit pathway was performed at each host site and included the stages of imaging, applicator reconstruction, treatment planning and delivery. Deviations between planned and measured dose distributions were quantified using gamma analysis methods. Local procedures were also discussed between auditors and hosts.

Results: The mean difference between Reference Air Kerma Rate (RAKR) measured during the audit and RAKR specified by the vendor source certificate was $1.3 \%$. The results of end-to-end tests showed a mean difference between calculated and measured dose of $2.5 \%$ with TLDs and less than $0.5 \%$ with Farmer chamber measurements. GafChromic films showed a mean gamma passing rates of $>95 \%$ for plastic and metal applicators with 2\%/1 mm global tolerance criteria.

Conclusions: The results of this audit indicate dosimetric consistency between centres. The 'end to end' dosimetry audit methodology for HDR brachytherapy has been successfully implemented in a multicentre environment, which included different models of Ir-192 sources and different treatment planning systems.

The ability to create a 3D-printed water-equivalent phantom customised to accurately position all three detector types simultaneously at controlled distances from the Ir-192 source under evaluation gives good reproducibility for end-to-end methodology.
\end{abstract}

\section{Introduction}

The system of radiation protection used throughout Ireland and the European Union is based on the recommendations of the International Commission for Radiological Protection (ICRP). This system is embodied in various European directives most notably the Basic Safety Standards (BSS) Council Directive 2013/59/Euratom. The BSS Directive was transposed into Irish legislation through two Statutory Instruments (S. I.): the Ionising Radiation Regulations (S.I. No. 30 of 2019) for the protection of workers and members of public and the Medical Exposure Regulations (S.I. No. 256 of 2018) for the radiation protection of patients. The BSS Directive is the main legal instrument dealing with the protection of patients undergoing diagnostic and therapeutic procedures using medical ionising radiation. The BSS Directive asserts that Member States shall ensure that: "all reasonable measures are taken to minimise the probability and magnitude of accidental or unintended exposure of individuals subject to medical exposure".

ICRP published the report no. 97: "Prevention of high dose rate brachytherapy accidents" [1] as a guide for radiation protection in high dose rate (HDR) brachytherapy. This document highlights three main aspects of practice:

\footnotetext{
* Corresponding author.

E-mail address: sarah.bassi@asufc.sanita.fvg.it (S. Bassi).
} 
- HDR techniques deliver a very high dose rate, $>12 \mathrm{~Gy} / \mathrm{min}$, with large dose per fraction, often $>5 \mathrm{~Gy}$, so errors in delivery can lead to under- or over-dosage with the potential for adverse clinical effects.

- $>500$ HDR accidents (including one death) have been reported along the entire chain of procedures from source packing to delivery of dose. Human error has been the prime cause of radiation incidents.

- Many accidents could have been prevented if staff had functional monitoring equipment and paid attention to the results.

Many errors have been reported in literature regarding brachytherapy delivery [2-6]. These include well chamber calibration error [2], confusion over units [3], and at least three incidents involving incorrect dwell positions [4-6]. Field safety notices from equipment manufacturers [7-9] describe other errors and potential errors related to HDR brachytherapy. Consequences of accidental incorrect exposure can be very severe and affect many patients. There have been media reports of some of these errors [4-6] which could affect public opinion and undermine public confidence in HDR brachytherapy as a treatment option.

A strong Quality Assurance program may not be enough to detect issues that are difficult to identify through the systematic Quality Controls and dosimetric audits. Inter-institution comparisons are important tools to assess the accuracy of radiation dose delivered and consistency between centres. In external beam radiotherapy (EBRT) dosimetric inter-hospital comparisons and audits are accepted as a way to assess consistency between centres and to detect systematic errors $[10,11]$. The objectives of audit in brachytherapy are similar to the ones of external beam radiotherapy: to detect systematic errors, to provide reassurance, to enable improvements, and to demonstrate compliance. In brachytherapy, a dosimetry system end-to-end audit may be useful to evaluate aspects of dosimetric accuracy. However, the audit process cannot uncover all potential sources of human error that could occur but gives reassurance that there is a robust system in place.

This paper presents a HDR dosimetric audit and an inter-hospital comparison that was performed in Ireland from February to July 2019. Prior to developing the dosimetry audit methodology, a review was conducted on journal articles published over the last decade, that have discussed concepts, research and practices of dosimetric audit in brachytherapy. Medline/PubMed and Embase databases were searched, internet search engines utilised and International Atomic Energy Agency recommendation [10] consulted. The methodology of National Brachytherapy audits from Palmer and Diez [12,13], Haworth [14], Tedgren [15] and Heeney [16] are taken into account.

In comparison to external beam radiotherapy, the process for dose calculation and treatment delivery in brachytherapy is relatively simple. In general, brachytherapy sources are supplied with manufacturers source strength certificates expressed to an accuracy of $5 \%(k=3)$ for high dose rate (HDR) sources and most HDR treatment planning systems (TPS) calculate dose based on the 1995 report of the AAPM Radiation Therapy Committee Task Group $43[17,18]$. However, this does not mean that dosimetric audit is without complexity. Indeed, the high dose gradients, orders of magnitude of variation in dose deposition across clinical regions of interest, and small spatial scales, mean that measurements to verify delivered dose and dose distribution are challenging. The performance of an HDR unit and its ability to accurately implement a series of planned source dwells is critical to the quality of the treatment. The accuracy of delivered doses is particularly dependent on source positioning due to short distances between target and source, steep dose gradients and large inverse square law corrections for any positioning inaccuracies.

\section{Materials and methods}

\subsection{Equipment}

In order to assess the consistency between HDR brachytherapy centres, a dosimetric audit and an inter-hospital comparison was performed at six departments on the island of Ireland. The objective of the audit was to investigate both the dosimetric and positional accuracy of HDR treatment units. The following systems combining HDR after-loaders and brachytherapy treatment planning systems were included in the audit:

- Nucletron microSelectron (MICROSELECTRON V2) and Oncentra ${ }^{\circledR}$ Brachy TPS, in use in three centres,

- Flexitron microSelectron (FLEXISOURCE Ir-192) and Oncentra ${ }^{\circledR}$ Brachy TPS in use in two centres,

- Gammamed Plus (IR-192 GAMMAMED PLUS HDR 0.9 MM) and BrachyVision TPS in use in one centre.

Firstly, the Ir-192 source certificate from each centre was checked as per the procedure described in section 2.2 (calibration check of sources). A 0.6 cc waterproof Farmer chamber (PTW TM30013) was used to calibrate the sources in terms of reference air kerma rates (RAKR). RAKR is defined by ICRU Report 38 [19] as the kerma rate to air corrected for air attenuation and scattering and is expressed in units of $\mathrm{mGy} / \mathrm{h}$. Correction factors proposed by Reynaert [20] were used to convert the RAKR to dose to water. This initial check, performed in each host department provides an independent check of the source calibration for the purposes of the audit.

Secondly, a 3D-printed water-equivalent phantom was created to position the PTW Farmer Chamber, a GafChromic EBT3 film and 2 Thermo Scientific ${ }^{\mathrm{TM}}$ TLD-100 Lithium Fluoride (LiF) thermoluminescent detectors (TLD). At each audited centre an end-to-end test was performed by the local staff using local clinical devices, under the supervision of auditors. A set of CT images of the 3D phantom with detectors and applicator in place was acquired. From this, the treatment plan was calculated using the clinical TPS and the treatment was delivered with clinical HDR afterloading device.

The chamber was used to measure the point dose predicted by the TPS and the GafChromic films were used to record the dose distribution for comparison with the dose distribution as calculated by the TPS. AAPM TG-43 $[17,18]$ recommends thermoluminescent detectors (TLD) as the gold standard dosimeter in brachytherapy. Nevertheless, the use of TLDs in a high dose gradient is quite challenging for determining point dose due to the large positioning inaccuracies compared to a Farmer Chamber. Hence, TLDs are used in this audit for a dual purpose: as an independent detector to simultaneously measure the delivered dose and as a means to compare with the published work of Palmer et al. [12].

The independent calibration of detectors and the measurement of calibration coefficient of Farmer Chamber is described in section 2.3 (calibration of detectors). Calibration of the chamber and TLDs was done in advance of the audits. At each host centre, the auditing team used the same Farmer chamber and provided the pre-calibrated TLD detectors.

The actual audit was performed using the custom 3D-printed phantom, and the test is described in section 2.4 (end-to-end test).

Finally, a checklist was designed following IAEA QUATRO [10] recommendations to help auditors to organise the audit program and to ensure coverage of all relevant topics.

There was a team of four physicists on the audit team of which two participated at each host site. Only one of the four audit physicists was present at all of the host-site audits. The host site being audited provided two local physicists to participate in the audit. In this way, the audit itself underwent technical improvement thanks to the experience of several auditors.

\subsection{Calibration check of sources}

To minimise errors in source calibration, an independent check of the source strength was performed using the "Kreiger" type full-scatter phantom [21,22,23]. The Farmer chamber was placed in this cylindrical PMMA phantom with well-described dimensions, at a known 
distance from the central needle in which the source travels (see Fig. 1). This phantom has four different positions around the source to locate the chamber, in order to compensate for the source anisotropy and scatter. Deutsche Gesellschaft fur Medizinische Physik (DGMP) Report 13 gives generic correction factors for converting the measured charge to RAKR [21].

\subsection{Calibration of detectors}

\subsubsection{The audit farmer chamber}

The Farmer Chamber was calibrated using the same "Krieger" dosimetry phantom described in section 2.2 and an Ir-192 calibrated source from GammaMedPlus (Varian, Palo Alto) brachytherapy afterloader. The Ir-192 source strength calibration certificate, provided by the supplier, was compared to a well type ionization chamber Secondary Standard measurement, following PTB code of practice for Ir-192 sources [24]. The difference between the RAKR declared value from the source certificate and the measured value using the Secondary Standard was $1 \%$. This calibrated source was used to calculate the Farmer Chamber calibration coefficient for Ir-192 to be used at each host centre during the audit.

This value for the Farmer chamber was checked against the NE2611B Secondary Standard photon chamber traceable to NPL for kilovoltage and megavoltage photon beams, following the IPEM recommendations for $\mathrm{kV}$ beams $[25,26]$ and the Code of Practice for MV beams [27]. The photon beams used for this calibration check were: $250 \mathrm{kV}$ from an Xstrahl 200 (Gulmay Medical) and 6 MV from a Siemens Oncor clinical Linac. The Ir-192 source is a gamma emitter with a wide energy spectrum and an average energy of $360 \mathrm{keV}$. The calibration factor was calculated at this energy by a linear interpolation using the Half Value Layer (HVL), between the calibrations at $250 \mathrm{kV}$ and $6 \mathrm{MV}$.

\subsubsection{GafChromic film}

In order to calibrate GafChromic EBT3-V3 films, an energy independence check was performed as published by Bassi et al. [28]. Film was irradiated at increasing doses with three different beams: $6 \mathrm{MV}$ beam from Elekta Precise Linac, $\mathrm{TPR}_{20,10}=0.684 \pm 0.01, \mathrm{HVL}=2.00 \pm$ $0.01 \mathrm{~mm} \mathrm{Al}$ and $\mathrm{HVL}=0.20 \pm 0.01 \mathrm{~mm} \mathrm{Al}$, from Xstrahl Gulmay Orthovoltage unit. The dose output for each beam was determined with an appropriate calibrated ionisation chamber, following IPEM [27] and DIN6809-4 [29] standards recommendations. Calibration curves were generated using the same dose range ( 0 cGy to $850 \mathrm{cGy}$ ) for the three energies. Using the $6 \mathrm{MV}$ calibration curve as reference, the film response in terms of net optical density (OD) was evaluated.

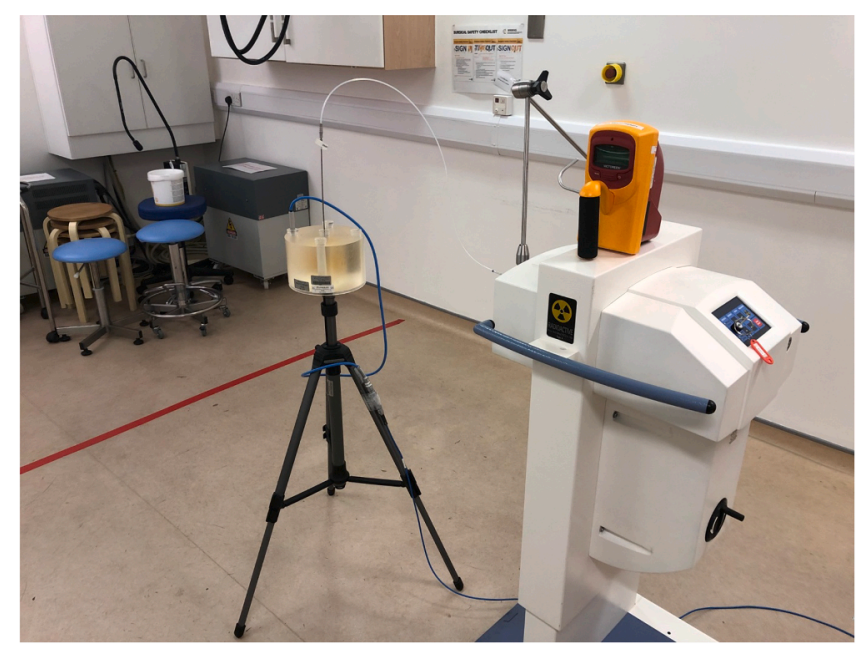

Fig. 1. Farmer chamber calibration with Ir-192 calibrated source from GammaMed Plus and Krieger phantom on tripod stand.

\subsubsection{TLD detectors}

Eight TLD-100 (LiF:Mg, Ti) square chips, with dimensions $3 \times 3 \times 1$ $\mathrm{mm}^{3}$, were calibrated separately. They were irradiated using a $6 \mathrm{MV}$ beam and $80 \mathrm{cGy}$ dose from a Siemens Oncor clinical Linac and were read out using a Thermo Scientific ${ }^{\mathrm{TM}}$ HARSHAW TLD ${ }^{\mathrm{TM}}$ Model 3500 Manual Reader. A glow curve was produced from the reading cycle which was related back to dose. This calibration process was done 3 times in total for all the chips, checking the constancy of readings. Before being irradiated, each TLD chip was annealed at $500{ }^{\circ} \mathrm{C}$ in an oven and cooled on to the ambient temperature. The whole process lasted $5 \mathrm{~h}$. After the reading, the TLD chip was again annealed and reused.

\subsection{End-to-end test}

The most common way to test a system workflow from start to end is to perform an end-to-end test.

\subsubsection{The 3-D audit phantom and detector set-up}

For this purpose, a custom phantom was designed to locate the Farmer Chamber, GafChromic EBT3 films and TLDs (see Fig. 2) at $2 \mathrm{~cm}$ distance from the source. Precisely cut holes were cut to rigidly fix the ionisation chamber and the source applicator holders (plastic and/or metallic needles) along the centre of the phantom. An AirWolf Axiom 20 $3 \mathrm{D}$ printer was used to print the 3D phantom with Cheetah ${ }^{\mathrm{TM}}$ (NinjaTek) filament [30] via Fused Deposition Modelling (FDM) 3D printing technology. The water equivalence of material at Ir-192 energies was assessed in a previous work [31]. The phantom was printed with $0.5 \mathrm{~mm}$ nozzle, $100 \%$ fill selected, $0.35 \mathrm{~mm}$ layer height, print temperature of $255^{\circ} \mathrm{C}$ and bed temperature of $40^{\circ} \mathrm{C}$, print speed $40 \mathrm{~mm} / \mathrm{s}$. Two dummy TLDs (markers) were positioned at $2 \mathrm{~cm}$ distance from the central axis of applicator on the phantom in position 1 and 2 (see Fig. 2). The position was marked with a cross. A CT scan was acquired and imported to the TPS of each centre. The optimal measurement position or "sweet point" of Farmer chamber was located using the 3D printed phantom, irradiating the chamber for $10 \mathrm{~s}$ at several dwell positions, using a step size of $2.5 \mathrm{~mm}$ to detect the position of maximum signal.

\subsubsection{The imaging stage}

A CT-scan, with $1 \mathrm{~mm}$ slice thickness, was acquired and the "sweet point" distance from the chamber tip was recorded $(1.02 \pm 0.2 \mathrm{~cm})$. Fig. 3 shows the "sweet point" location in the coronal view. The effective point of measurement in the trans-axial plane is at 0.5 times the radius from the tip of Farmer Chamber (radius $=3 \mathrm{~mm}$ as reported by PTW's manual).

\subsubsection{The planning stage}

An external structure and TLD structures were contoured on the CT image set. The applicator was reconstructed and a plan was created to deliver uniform dose across the two TLDs, dose ranging between 60 and 90 cGy. In order to obtain a uniform dose across the plane of each TLD, the plan was created using at least 20 weighted dwell positions, at $5 \mathrm{~mm}$ step size, to shape a line source dose distribution. In this way, the dose variation in the TLD chip was related only to distance from the source, due to the high dose gradient. The TLDs were irradiated with the planned dose and the measurements were repeated at least twice. All the measurements were performed in a small water tank phantom (see

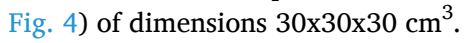

A second plan was created to irradiate the films with a dose in the range between 700 and $900 \mathrm{cGy}$. The range was chosen in order to avoid the dose dependence of the film. The plan was similar to the previous one: a line source dose distribution was created using several wedged dwell positions. At least 2 films in each centre were irradiated, the plan was delivered at least twice, and the reproducibility of plan delivery was tested. Irradiated films were scanned with an Epson Expression 10,000 XL scanner, at 150 DPI spatial resolution. Dose distributions were acquired using Film QA Pro software and were compared with TPS 


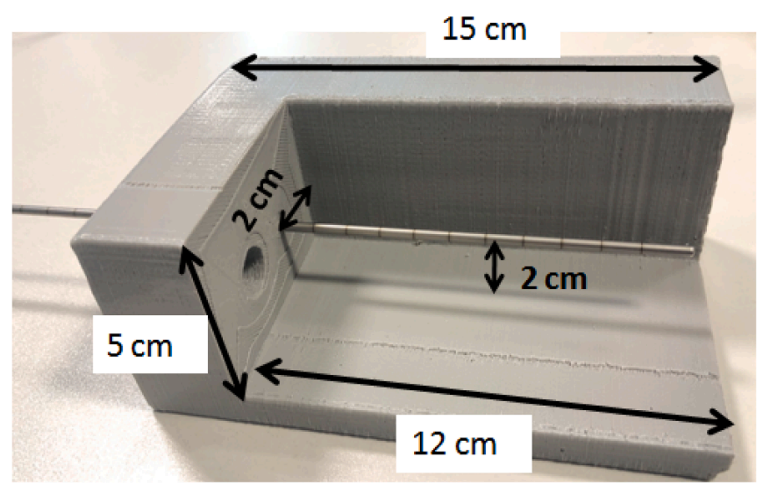

(a)

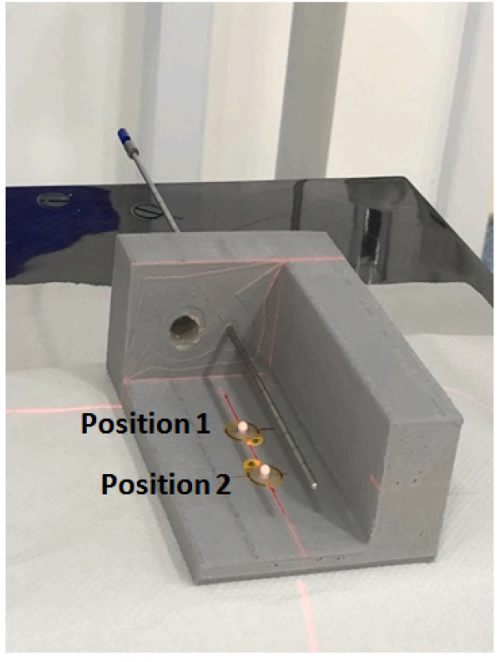

(b)

Fig. 2. (a) 3D printed phantom created to place the GafChromic EBT3 films and the TLDs detectors; (b) 3D printed phantom with metallic needle for source holding and dummy TLDs (markers) for the location on CT scan. The position is marked by a red cross drawn down the phantom. The source travels vertically through the applicator needle. (For interpretation of the references to colour in this figure legend, the reader is referred to the web version of this article.)

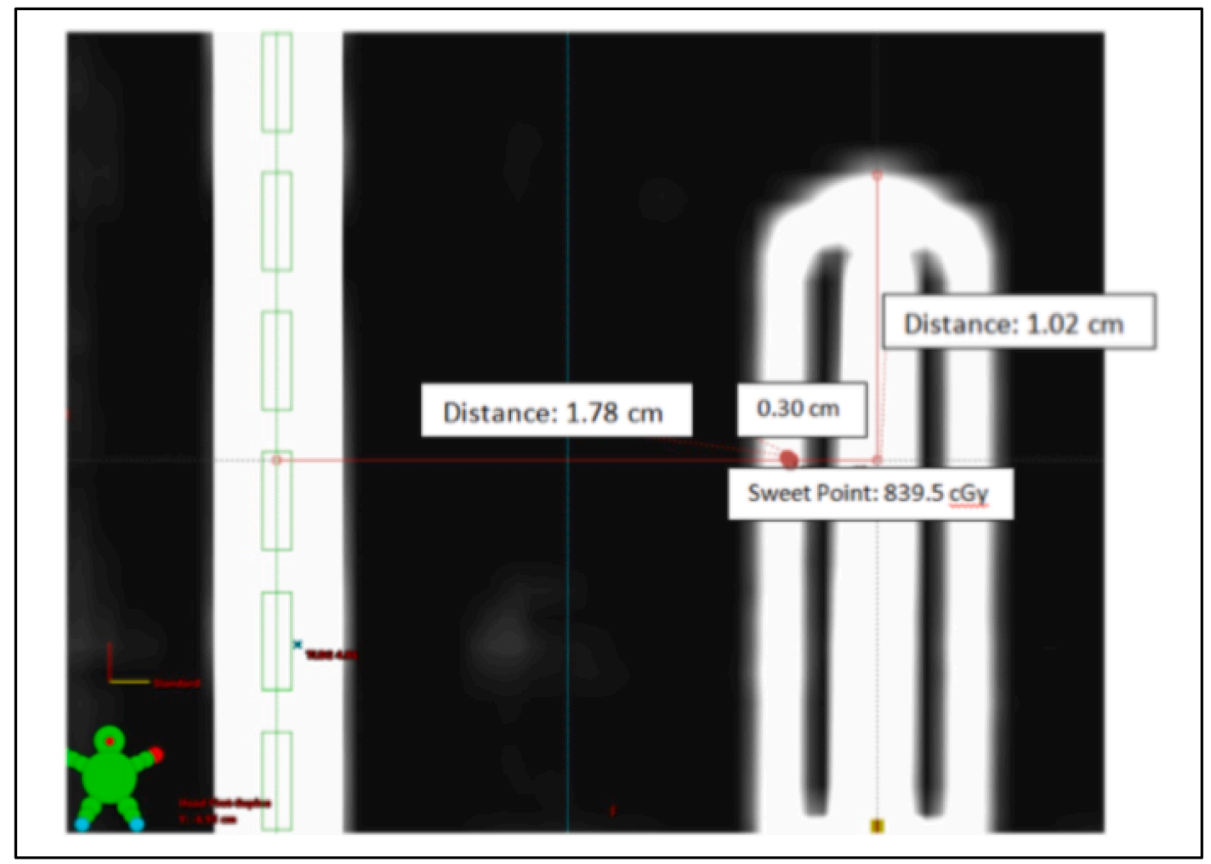

Fig. 3. Sweet point position from CT scan. The figure shows the distance between source and sweet point $(1.78 \mathrm{~cm})$ in transaxial plane and the distance between chamber tip and sweet point $(1.02 \mathrm{~cm})$. The radius of chamber is $3 \mathrm{~mm}$.

calculations. Gamma analysis was repeated for the same film in order to ensure pass rate reproducibility. Oncentra ${ }^{\circledR B}$ Brachy TPS allows export of a $3 \mathrm{D}$ cubic dose grid that is a sub-matrix of the whole calculated plan dose matrix. The sub-matrix can be located in any position of CT image set. Using this feature, the dose calculated by TPS in the region where the film is located can be exported and compared with measured film dose, using Film QA Pro software. This procedure was adopted in the 5 centres where Oncentra ${ }^{\circledR}$ Brachy was in clinical use.

A third plan was created in order to irradiate the sweet point of Farmer chamber using a point source geometry with dose ranging between 700 and $900 \mathrm{cGy}$. Dose at the sweet point and at $\pm 0.5 \mathrm{~mm}$ distance from the sweet point were recorded and compared with the chamber readings. This spatial tolerance was introduced to take into account the positioning uncertainties of Farmer Chamber and the $1 \mathrm{~mm}^{3}$ voxel dose matrix of the TPS. At least 3 readings were recorded. The Farmer chamber was placed in a small water tank with its axis vertical, (see Fig. 4) and the measured current (nC) was converted to air-kerma in water using the calibration factor and the pressure and temperature correction factors. To convert the measured air-kerma in water to dose to water a conversion factor (CF) provided by N. Reynaert [20].

\subsection{Uncertainties and tolerances}

The estimation of uncertainties in the measurements was related to the tolerances of tests undertaken. The use of different detectors provides the possibility to check the weaknesses of system, in order to discern the sources of errors. Multiple measurement devices create independence from a single detector. For every test a different tolerance is 


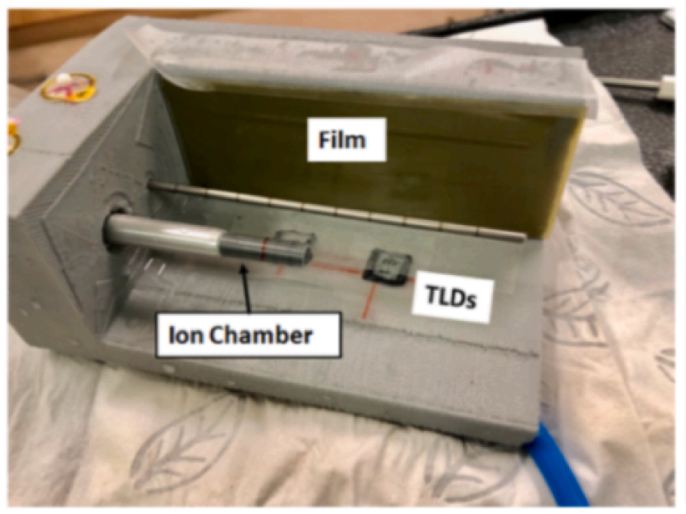

(a)

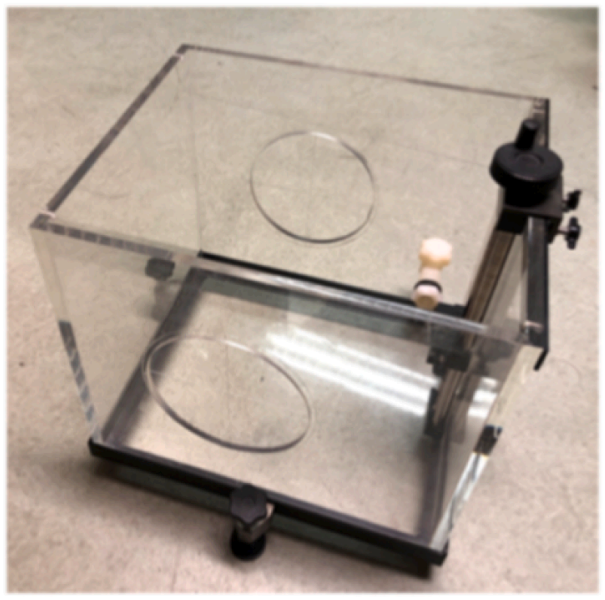

(b)

Fig. 4. (a) $3 D$ phantom with Ion chamber, TLD and films positioned and (b) small water tank $\left(30 \times 30 \times 30 \mathrm{~cm}^{3}\right)$ where the phantom was inserted, The Farmer chamber and applicator needle were placed vertically in the tank during the irradiation in order to reduce the sag of plastic applicator.

adopted, based on the measurement uncertainty of the detector itself.

\subsubsection{Well chamber}

The uncertainty from the Secondary Standard Well Type Chamber calibration certificate was quoted as $0.8 \%(\mathrm{k}=2)$ and the $\operatorname{Ir}^{192}$ source calibration certificate has a quoted uncertainty of $5 \%(\mathrm{k}=3)$. Therefore the source calibration test tolerances were set to $5 \%$. If the difference between measured and declared RAKR was out of tolerance, the audit could not be completed until the differences have been investigated and resolved.

\subsubsection{Farmer chamber}

The uncertainty in the calibration coefficient of NE2611 secondary standard, stated by the calibration certificate was $1.4 \%, \mathrm{CL}=95 \%(\mathrm{k}=$ 2 ). The same uncertainty was applied to the Farmer chamber, plus the uncertainty from the setup (temperature, pressure, polarity and recombination correction). The final uncertainty calculated using the Law of Propagation of Uncertainty [32] for the Farmer Chamber was $1.5 \%(\mathrm{k}=2)$. For the end-to-end test the Farmer chamber tolerances were: $1.5 \%$ in dose, due to the uncertainty in the calibration coefficient and setup and $0.5 \mathrm{~mm}$ of distance to agreement (DTA), taking in account the positioning uncertainties of the Farmer Chamber and the $1 \mathrm{~mm}^{3}$ voxel of TPS dose matrix. If the tolerance was exceeded during the audit an investigation was requested.

\subsubsection{GafChromic film}

Using a 3D dose map, the GafChromic films were compared with the dose calculated by TPS with FilmQA Pro. Gamma analysis [33] was implemented to quantify the level of agreement, with criteria $2 \% / 0.5$ $\mathrm{mm}$, using globally normalised doses. These tolerances were chosen because at $2 \mathrm{~cm}$ distance from the source a $0.5 \mathrm{~mm}$ distance to agreement parameter results in a $4 \%$ dose difference due to the steep dose gradient. Adding a $2 \%$ dose difference results in a $6 \%$ dose tolerance, which is comparable with the uncertainty proposed by AAPM TG-43 $[17,18]$ for dose calculation at these distances $(5.7 \%$ at $1 \mathrm{~cm}$ and $7.3 \%$ at $5 \mathrm{~cm}$ ). There are no recommendations about limits in minimum pass rate in brachytherapy. AAPM TG-218 publication recommends an action limit for intensity modulated radiation therapy (IMRT) when the pass rate is lower than $90 \%$, with $3 \% / 2 \mathrm{~mm}$ criteria and a $10 \%$ dose threshold [34]. Tighter tolerances are suggested in stereotactic radiosurgery (SRS) and stereotactic body radiotherapy (SBRT) cases [34]. Taking in consideration these recommendations, a reasonable tolerance limit adopted in the audit was a gamma pass rate $\geq 90 \%$, with $2 \% / 0.5$ $\mathrm{mm}$ criteria. If the tolerance was exceeded during the audit an investigation into the source positioning and CT scan was carried out.

\subsubsection{TLD}

The uncertainties for the TLD measurements were calculated using both AAPM TG-43 $[17,18]$ recommendations and the work of Rouè et al. [35]. The uncertainty associated with the LiF energy correction for Ir192 sources is reported by the work of Rouè and is $2.56 \%(\mathrm{k}=2)$. The total type B systematic uncertainties combined is $5.5 \%(\mathrm{k}=2)$. The tolerance limit for TLD measurements was set at 5.5\% and if exceeded during the audit an investigation was carried out. Table 1 summaries the uncertainties and tolerances implemented.

\section{Results}

\subsection{Calibration of detectors}

\subsubsection{Farmer chamber}

The Farmer Chamber calibration factor was measured at the auditors' department before the on site audits. The chamber was used both to check the Ir-192 source calibration factor of each host centre using the custom phantom, with $1.5 \%$ of uncertainty, $\mathrm{CL}=95 \%$. The reported RAKR from the calibration certificate was $3.206 \mathrm{cGy}^{*} \mathrm{~h}^{-1}$ and the RAKR measured using the well-type chamber was $3.117 \mathrm{cGy}^{*} \mathrm{~h}^{-1}$ (a difference of $2.8 \%$ ). The calibration coefficient of the Farmer Chamber was obtained using the Ir-192 source RAKR as measured using the Well Type Chamber. The second check of the Farmer Chamber was against NPL type 2611B chamber using the $250 \mathrm{kV}$ Orthovoltage beam and $6 \mathrm{MV}$ beam. The calibration coefficient at $360 \mathrm{kV}$ was calculated by liner interpolation between values obtained using the $250 \mathrm{kV}$ and $6 \mathrm{MV}$ photons beams based on the HVL of these beams. A Sr-90 constancy check was done to ensure stability of the secondary standard chamber.

The results of the cross calibration measurements between the Farmer chamber and the Secondary Standard chamber traceable to NPL at each photon energy, are given below:

Calibration factor at $250 \mathrm{kV}: 4.654 * 10^{7} \mathrm{~Gy} / \mathrm{C}$

Calibration factor at $6 \mathrm{MV}: 5.146 * 10^{7} \mathrm{~Gy} / \mathrm{C}$

Interpolation at $360 \mathrm{kV}: 4.697 * 10^{7} \mathrm{~Gy} / \mathrm{C}$

Calibration factor measured with Krieger phantom: $4.727 * 10^{7} \mathrm{~Gy} / \mathrm{C}$

Difference between interpolated/measured: $0.6 \%$

Further details of procedure and measurements are reported on Appendix.

\subsubsection{GafChromic film}

Using the $6 \mathrm{MV}$ calibration curve as a reference, the film response in 
Table 1

The table shows a list of uncertainties of dose measurements and the tolerances related to each test within the end-to-end pathway.

\begin{tabular}{|c|c|c|c|c|}
\hline Test & Detector & Uncertainty & Tolerance & Reference \\
\hline \multirow[t]{2}{*}{ Source calibration verification } & Farmer Chamber & $1.5 \%$ & $5 \%$ & Secondary Standard Calibration Certificate \\
\hline & & $\mathrm{k}=2$ & & Calibration Certificate of source \\
\hline \multirow[t]{6}{*}{ End-to-End } & TLD & $5.5 \%$ & $5.5 \%$ & AAPM TG-43 $[17,18]$ \\
\hline & & $\mathrm{k}=2$ & & Rouè et al [35] \\
\hline & Farmer Chamber & $1.5 \%$ & $1.5 \%, \pm 0.5 \mathrm{~mm}$ DTA & Calibration coefficient and TPS dose resolution \\
\hline & & $\mathrm{k}=2$ & & \\
\hline & Film & $2 \%$ & $2 \%, \pm 0.5 \mathrm{~mm}$ DTA & Calibration coefficient, AAPM TG-43 [17] and AAPM TG-218 [34] recommendation \\
\hline & & $\mathrm{k}=2$ & & \\
\hline
\end{tabular}

terms of net optical density (OD) was evaluated. The results showed that when GafChromic EBT3-V3 film was irradiated at doses higher than 500 cGy, the calibration curve for the $2 \mathrm{~mm}$ Al kilovoltage beam agrees with the reference beam $(6 \mathrm{MV})$ within $3 \%(\mathrm{k}=2)$. Hence, EBT3-V3 films are suitable dose detectors when the dose response curve is measured within the range $6 \mathrm{MV}$ and $2 \mathrm{~mm} \mathrm{Al} \mathrm{HVL}$. $6 \mathrm{MV}$ calibration curve could be used within this range, with $3 \%, \mathrm{k}=2$, uncertainty [28].

\subsection{Calibration of Ir-192 sources}

At each host brachytherapy centre, the RAKR of calibration certificate was compared with the RAKR measured using the "Krieger" type phantom and the auditor's Farmer Chamber. Table 2 shows the difference between certificate value of RAKR and the measured RAKR value obtained during the audit. All the values were within tolerances of $\pm 5 \%$. A comparison between the differences obtained at each host centre during the audit against the determination of the RAKR of the host source using the host's own secondary standard chamber (SS) is displayed in the last column of Table 2. In this case, the RAKR was measured with the host's own Secondary Standard Well-type Chamber of each centre.

\subsection{End-to-end test}

\subsubsection{TLD measurement results}

Differences between dose measured using TLDs and dose calculated by the host centre's TPS are reported on Table 3. In all centres the TLD test passed with a tolerance of 5.5\%, except for point 2 of centre 4 . In this case, the dose measured by 2 TLDs in this point was $13 \%$ different from planned dose.

Some centres used a plastic applicator needle compatible with their HDR afterloader while other centres used a metal needle applicator. The vertical positioning of the applicator needle in the audit phantom was designed to minimise any warping of plastic needles within the phantom due to the flexible nature of the plastic needles.

\subsubsection{Film pass rate results}

At least two films were used to check the dose distribution of the plan delivered, in each host centre. For each irradiation, only one film was used, so, the plan was delivered at least twice. In this way, the reproducibility of plan delivery was also tested. Table 4 reports the gamma pass rate with a tolerance $2 \% / 1 \mathrm{~mm}$ and Table 5 with $2 \% / 0.5 \mathrm{~mm}$ tolerance. The lower pass rates are highlighted in red font. The pass rate
Table 3

Comparison between dose measured by TLDs and calculated by TPS (positive difference means the measured dose is higher than the calculated).

\begin{tabular}{lllll}
\hline & Position & Measured (cGy) & Calculated (cGy) & Difference \\
\hline Centre 1 & $1\left(^{*}\right)$ & $69.7 \pm 1.7$ & 68.2 & $2.2 \%$ \\
& $2\left(^{*}\right)$ & $75.7 \pm 1.6$ & 74.5 & $1.6 \%$ \\
& 1 & $85.5 \pm 5.0$ & 89.9 & $-4.8 \%$ \\
Centre 2 & 2 & $91.9 \pm 2.3$ & 94.3 & $-2.6 \%$ \\
& 1 & $79.2 \pm 1.9$ & 80.8 & $-1.9 \%$ \\
Centre 3 & 2 & $79.4 \pm 1.6$ & 80.4 & $-1.3 \%$ \\
& 2 & $76.6 \pm 0.1$ & 78.7 & $-2.6 \%$ \\
Centre 4 & $1\left(^{*}\right)$ & $78.2 \pm 0.1$ & 81.3 & $-3.8 \%$ \\
& $2\left(^{*}\right)$ & $78.3 \pm 0.2$ & 80.4 & $-2.6 \%$ \\
Centre 5 & $1\left(^{*}\right)$ & $82.3 \pm 0.2$ & 81.0 & $-13 \%$ \\
& $2\left(^{*}\right)$ & $86.6 \pm 2.2$ & 85.2 & -3.4 \\
Centre 6 & $1\left(^{*}\right)$ & $77.8 \pm 3.8$ & 88.5 & -2.2 \\
& $2\left(^{*}\right)$ & $78.5 \pm 1.1$ & 81.6 & -2.2 \\
\hline
\end{tabular}

(*) Plastic needle.

for each centre was in tolerance.

Fig. 5 shows an example of dose distribution recorded by the GafChromic films. In the film inner area, where the dose gradient is steeper, the gamma value is $<1$ with $2 \% / 0.5 \mathrm{~mm}$ of tolerance. The more complex the dose distribution becomes, the more challenging it is to achieve high pass rates from gamma analysis of GafChromic film.

\subsubsection{Farmer chamber measurement results}

Table 6 shows the comparison between the dose measured by the Farmer Chamber and the dose calculated by host centre's TPS. The Farmer Chamber readings were corrected for the calibration coefficient (measured using "Kreiger" phantom), temperature and pressure coefficient, ion recombination coefficient, polarity coefficient and air-kerma in water to dose to water conversion factor $(\mathrm{CF})$ provided by N. Reynaert [18]. The last factor depends on the distance between the detector and the source. Small differences (less than $2 \%$ ), measured on the CT image set acquired at each host centre, were observed between centres due to the small differences in the Farmer Chamber positioning within the $3 \mathrm{D}$ phantom.

\section{Discussion}

An 'End-to-end' audit with a customized 3D-printable phantom has been successfully performed in six Irish brachytherapy departments. The

Table 2

RAKR comparisons between measured and certificate value (positive difference means the measured RAKR is higher than the certificate value). The last column, labelled as "difference against SS on site", shows the differences with the calibration certificate value obtained by each host centre during the source exchange, using the host's own Secondary Standard Well Type Chamber as reference detector.

\begin{tabular}{|c|c|c|c|c|c|c|}
\hline & Measure (nC) & $\mathrm{Kt}, \mathrm{p}$ & Measured RAKR (cGy/h) & Certified RAKR (cGy/h) & Difference (\%) & Difference against SS on site (\%) \\
\hline Centre 1 & $1.441 \pm 0.024$ & 1.009 & $3.117 \pm 0.052$ & $3.206 \pm 0.160$ & $2.8 \%$ & $1.7 \%$ \\
\hline Centre 2 & $1.453 \pm 0.014$ & 0.995 & $3.099 \pm 0.029$ & $3.156 \pm 0.158$ & $1.8 \%$ & $0.1 \%$ \\
\hline Centre 3 & $1.236 \pm 0.033$ & 1.033 & $2.738 \pm 0.074$ & $2.734 \pm 0.137$ & $-0.2 \%$ & $-0.17 \%$ \\
\hline Centre 4 & $1.532 \pm 0.069$ & 1.004 & $3.300 \pm 0.015$ & $3.245 \pm 0.162$ & $1.7 \%$ & - \\
\hline Centre 5 & $1.692 \pm 0.021$ & 1.007 & $3.654 \pm 0.046$ & $3.654 \pm 0.183$ & $0.0 \%$ & - \\
\hline
\end{tabular}


Table 4

GafChromic film pass rate with $2 \% / 1 \mathrm{~mm}$ tolerance, in red the lower pass rate.

\begin{tabular}{|c|c|c|c|c|c|c|}
\hline Film & Centre 1 & Centre 2 & Centre 3 & Centre 4 & Centre 5 & Centre 6 \\
\hline $\mathbf{1}$ & $99.7 \%$ & $100 \%$ & $100 \%$ & $100 \%$ & $99.8 \%$ & $98.2 \%$ \\
\hline $\mathbf{2}$ & $100 \%$ & $99.95 \%$ & $99.97 \%$ & $99.36 \%$ & $98.7 \%$ & $96.8 \%$ \\
\hline $\mathbf{3}$ & $99.94 \%$ & $99.77 \%$ & & & $100 \%$ & $97.8 \%$ \\
\hline
\end{tabular}

Table 5

GafChromic film pass rate with $2 \% / 0.5 \mathrm{~mm}$ tolerance, in red the lower pass rate.

\begin{tabular}{|c|c|c|c|c|c|c|}
\hline Film & Centre 1 & Centre 2 & Centre 3 & Centre 4 & Centre 5 & Centre 6 \\
\hline $\mathbf{1}$ & $97.2 \%$ & $99.6 \%$ & $97.78 \%$ & $99.9 \%$ & $96 \%$ & $92.3 \%$ \\
\hline $\mathbf{2}$ & $94 \%$ & $99.25 \%$ & $93.64 \%$ & $95.2 \%$ & $94 \%$ & $93.6 \%$ \\
\hline $\mathbf{3}$ & $98.75 \%$ & $96.39 \%$ & & & $99.6 \%$ & $93.1 \%$ \\
\hline
\end{tabular}
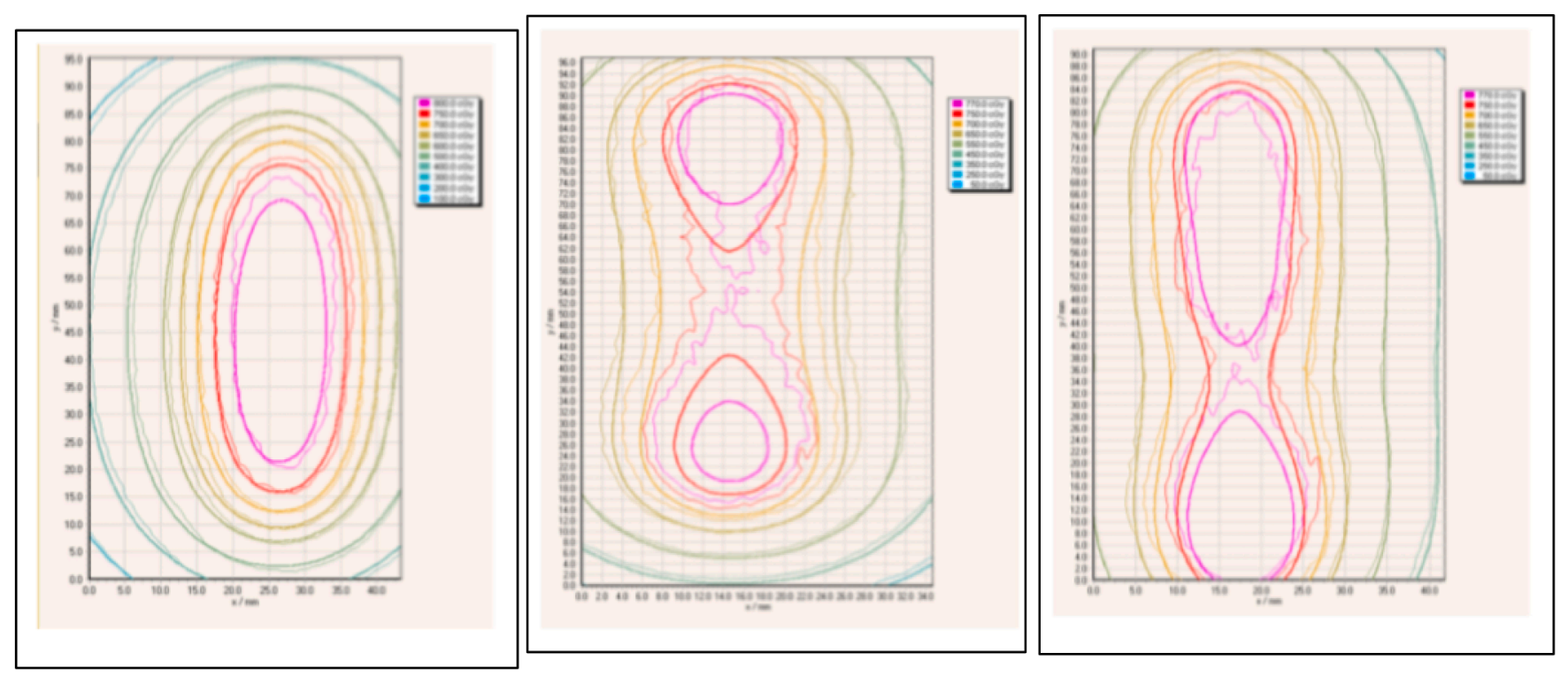

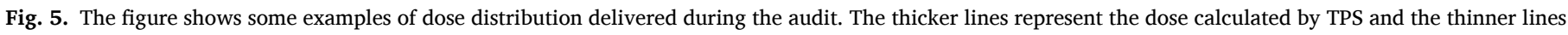
the dose measured by the film. The pass rate is $100 \%$ with $2 \% / 2 \mathrm{~mm}$ in each centre.

results obtained in this audit and the reproducible workflow of the audit tests performed demonstrate that $3 \mathrm{D}$ printable materials can be used for Ir-192 afterloading dosimetric audits and inter-hospital comparisons.

The innovative part of 3D printable materials is the ability for customisation: the phantom presented in this work is quite simple but centres can request a 3D phantom designed for a specific clinical application of their department and remotely perform an end-to-end test using the method described in this paper. Compared with traditional technique that involves the use of blocks of water equivalent phantom, $3 \mathrm{D}$ phantoms have the potential for greater accuracy because the customisation allows the checking of dose points and dose distributions specific to the clinical treatment.

Excellent agreement was seen between film-measured and TPS calculated isodose distributions. The passing rates from gamma analysis techniques were: $>90 \%$ with $2 \%$ dose and $0.5 \mathrm{~mm}$ DTA, $>95 \%$ with $2 \%$ $/ 1 \mathrm{~mm}$ and $100 \%$ with $2 \% / 2 \mathrm{~mm}$.

TLDs are challenging to use because, due to their size, the position is difficult to reproduce on a phantom. In all centres the TLD test was passed with a tolerance of $5.5 \%$, except for centre 4 . In this case, the dose measured by TLDs in a point was $13 \%$ different from the planned dose. This difference could be most likely attributed to a position error rather than a TLD reading and/or calibration error, because both TLDs gave the same result, suggesting a systematic error, as a shift of measurement position. A different 3D phantom design, with the creation of TLDs dedicated inserts, could help remove the error. The results of the Haworth at al [15] audit reported TLD results which fell within 5\% which is similar to this work.

The TLDs measurements reported in this work show an average 
Table 6

Comparisons between dose measured by the Farmer Chamber in the audit phantom and calculated by TPS in each centre. The mean difference is less than $0.5 \%$ and all the values are within the $1.5 \%$ in dose and $\pm 0.5 \mathrm{~mm}$ of DTA.

\begin{tabular}{|c|c|c|c|c|c|}
\hline & $\begin{array}{l}\text { Measure } \\
(\mathrm{nC})\end{array}$ & $\mathrm{CF}$ & $\begin{array}{l}\text { Dose } \\
\text { measured } \\
\text { (cGy) }\end{array}$ & $\begin{array}{l}\text { Dose } \\
\text { calculated } \\
\text { (cGy) }\end{array}$ & $\begin{array}{l}\text { Difference } \\
(\%)\end{array}$ \\
\hline $\begin{array}{c}\text { Centre } \\
1\end{array}$ & $\begin{array}{l}139.5 \pm \\
0.1\end{array}$ & 1.30 & $839.3 \pm 0.7$ & 839.5 & $-0.03 \%$ \\
\hline $\begin{array}{c}\text { Centre } \\
2\end{array}$ & $\begin{array}{l}134.9 \pm \\
0.2\end{array}$ & 1.30 & $831.2 \pm 1.1$ & 839.5 & $-1.2 \%$ \\
\hline $\begin{array}{c}\text { Centre } \\
3\end{array}$ & $\begin{array}{l}147.7 \pm \\
0.9\end{array}$ & 1.31 & $926.6 \pm 8.6$ & 920.9 & $0.6 \%$ \\
\hline $\begin{array}{c}\text { Centre } \\
4\end{array}$ & $\begin{array}{l}147.5 \pm \\
0.3\end{array}$ & 1.33 & $928.7 \pm 1.8$ & 924.3 & $-0.47 \%$ \\
\hline $\begin{array}{c}\text { Centre } \\
5\end{array}$ & $\begin{array}{l}133.8 \pm \\
0.7\end{array}$ & 1.27 & $808.5 \pm 4.2$ & 807.4 & $-0.14 \%$ \\
\hline
\end{tabular}

difference for the plastic applicators of $-1.1 \%(2.4 \% \mathrm{sd})$ and for the metal applicators of $-2.8 \%$ (1.3\% sd). Palmer et al. [12] reports an average difference for the plastic applicators of $-0.6 \%(1.3 \% \mathrm{sd})$ and for the metal applicators of $-3.0 \%(1.3 \% \mathrm{sd})$ using GafChromic films as detectors. The comparison between the results of this work with Palmer et al. work provides reassurance in the outcome of this audit.

An innovative part of this audit work was to use three very different detectors; Farmer chamber, GafChromic film and TLD detectors during the audit process at each host centre. Although the treatment plan for irradiating the GafChromic film differed from plan used to irradiate the TLDs due to differences in the dose appropriate for calibration conditions, the Farmer chamber measurement was obtainable during all irradiations. Hence for each treatment delivery at least two detectors were recording dose simultaneously.

The audit is also notable for comparing the expected reference air kerma strength of each Ir-192 source from the following three processes:

- The vendor certificate value of RAKR for the Ir-192 source

- The host centre measurement of the RAKR of the IR-192 source using the host's secondary standard well chamber

- The auditor's measurement of the RAKR of the host's IR-192 source using a Krieger phantom.

The value of the RAKR used within the TPS at each centre under audit is the value determined by the source certificate but this audit shows the variation in source RAKR when other methods are used.

Future improvements would include refinement of the audit phantom design to create an insert to localise the TLDs more precisely and to provide an easier method for placing the detectors without moving the needle or the Farmer chamber.

\section{Conclusions}

This paper represents both a novel approach to the study of 3D phantoms, which are implemented with success in a multicentre environment, and it could be an example for future audits, where different models of Ir-192 sources and different treatment planning systems have to be tested. The paper has described an end-to-end methodology for performing a dosimetric audit for Ir-192 HDR brachytherapy treatment pathways at multiple radiotherapy institutions. The pathway interrogates the process from imaging and treatment planning through to dose delivery. Three different types of radiation detectors are used to analyse and quantify the dose delivered using the HDR brachytherapy after-loading systems compared to the planned dose calculated by the HDR treatment planning system.

The methodology involved a purpose built 3D printable phantom that has allowed good reproducibility and constancy of approach at each of the six centres which participated in the audit. The results indicate dosimetric consistency between the centres. Further focused studies could aim at creating of a $3 \mathrm{D}$ phantom specific design to localize the TLDs with more reproducibility.

Brachytherapy audits are less common than external beam radiotherapy ones and the customization is generally rare. 3D printers have the advantage to create phantoms that are customized for the needs of each brachytherapy department and for this reason the use of them is encouraged. Hence, we hope that users may benefit from this study as a guidance both for future HDR brachytherapy audits and for the implementation of 3D phantoms.

\section{Appendix A. Supplementary data}

Supplementary data to this article can be found online at https://doi. org/10.1016/j.ejmp.2020.10.005.

\section{References}

[1] ICRP Publication 97. Prevention of high-dose-rate brachytherapy accidents. Ann. ICRP. 2005a;35(2).

[2] Lessons learned from a HDR brachytherapy well ionisation chamber calibration error. Available from: ResearchGate.

[3] USNRC. United States Nuclear Regulatory Commission. NRC Information Notice 2009-17: Reportable medical events involving treatment delivery errors caused by confusion of units for the specification of brachytherapy sources. Washington: NRC; 2009. Available from: https://www.nrc.gov/docs/ML0807/ML080710054. pdf.

[4] Sydney Morning Herald. Cancer therapy missed tumour sites. 21 June 2003a. Available from SydneyMorningHerald Sydney Morning Herald.

[5] Critically ill received wrong radiation therapy due to human errors: review. 12 September 2003b. Available from SMH/.

[6] The Hamilton Spectator (02/01/2019): Mistargeted radiation feared in 25 cases at Hamilton's Juravinski Centre. Available from: https://www.thespec.com/newsstory/9106915-mistargeted-radiation-feared-in-25-cases-at-hamilton-s-juravinskicancer-centre.

[7] Nucletron. Urgent field safety notice: Flexitron HDR. Reference 555.00267FSN. 2014 [cited 17 Sept 2014, 2p]. Available from: http://www.mhra.gov.uk/home/ groups/fsn/documents/fieldsafetynotice/con249618.pdf.

[8] Varian Medical Systems. Urgent field safety notice: Varisource iX console incorrect dwell times under special circumstances. Varian document PNL-VS-02.2010. April 2010. [cited 17 Sept 2014, 5p]. Available from: MHRA.

[9] Eckert \& Ziegler BEBIG GmbH. Urgent Field Safety Notice reference 2014LCT82. Mispositioning [sic] of source. Eckert \& Ziegler Bebig GmbH, Berlin, Germany, 17 March 2014.

[10] Quality Assurance Team for Radiation Oncology (QUATRO) IAEA - Comprehensive Audits of Radiotherapy Practices: A Tool for Quality Improvement - Vienna 2007.

[11] Physics aspects of safety assurance in high dose rate brachytherapy: quality control testing and implementation of dosimetry audit | Request PDF. Available from: ResearchGate.

[12] Palmer AL, Diez P, Gandon L, Wynn-Jones A, Bownes P, Lee C, Aird E, Bidmead M, Lowe G, Bradley D, Nisbet A. A multicentre 'end to end' dosimetry audit for cervix HDR brachytherapy treatment. Radiother Oncol 2015;114(2):264-71. Copyright (C) 2015 Elsevier Ireland Ltd. Available from: ClinicalKey.

[13] Diez P, Aird E, Gouldstone C, Sander T, Eaton D, Sharpe P. OC-0271: A multicentre audit of HDR and PDR brachytherapy absolute dosimetry in association with the INTERLACE trial. Radiother Oncol 2015;115:S139.

[14] Haworth A, Wilfert L, Butler D, Ebert MA, Todd S, Bucci J, et al. Australasian brachytherapy dosimetry audit. J Med Imag Radiat Oncol 2013;57:490-8. https:// doi.org/10.1111/1754-9485.12042.

[15] Tedgren Åsa Carlsson, Grindborg Jan-Erick. Audit on source strength determination for HDR and PDR 192Ir brachytherapy in Sweden. Radiother Oncol 2008;86(1):126-30. https://doi.org/10.1016/j.radonc.2007.12.008.

[16] Heeney C, McClean B, Kelly C. A dosimetric intercomparison of brachytherapy facilities in Ireland, Scotland and the North of England. Radiother Oncol 2005;74 (2):149-56.

[17] Rivard M J, Coursey B M, et al. Update of AAPM Task Group No. 43 Report: A revised AAPM protocol for brachytherapy dose calculations, AAPM report, 2004 [AAPM].

[18] Rivard M, Butler W, DeWerd L, Huq M, Ibbott G, Meigooni A, Melhus C, Mitch M, Nath R, Williamson J. Supplement to the 2004 update of the AAPM Task Group No. 43 Report. Med Phys 2007;34(6Part1):2187-205.

[19] ICRU (International Commission on Radiation Units and measurements). Dose and volume specification for reporting intracavitary therapy in Gynaecology-ICRU Report no. 38; 1985.

[20] Reynaert N, Verhaegen F, Thierens H. In-water calibration of PDR192Ir brachytherapy sources with an NE2571 ionization chamber. Phys Med Biol 1998; 43(8):2095-107.

[21] Krieger H and Baltas D 1999 Praktische Dosimetrie in der HDR-Brachytherapie Report No. 13 of DGMP (Deutsche Gesellschaft fur Medizinische Physik) (in German), referenced in Venselaar and Perez-Calatayud (2004). 
[22] Roué A, Venselaar J, Ferreira I, Bridier A, Van Dam J. Development of a TLD mailed system for remote dosimetry audit for 192Ir HDR and PDR sources. Radiother Oncol 2007;83(1):86-93.

[23] Pérez-Calatayud J, Granero D, Ballester F. Phantom size in brachytherapy source dosimetric studies. Med Phys 2004:31(7):2075-81.

[24] Selbach H-J. Neue Kalibrieranlage fur" 192Ir- und 60Co-Brachytherapie-Strahlungsquellen Tagungsband der 37. Jahrestagung der Deutschen Gesellschaft fur Medizinische. Physik (DGMP), Regensburg 2006:244.

[25] Klevenhagen SC, Aukett RJ, Harrison RM, Moretti C, Nahum AE, Rosser KE. The IPEMB code of practice for the determination of absorbed dose for x-rays below $300 \mathrm{kV}$ generating potential $(0.035 \mathrm{~mm} \mathrm{Al}-4 \mathrm{~mm}$ Cu HVL; $10-300 \mathrm{kV}$ generating potential). Phys Med Biol 1996;41:2605-25.

[26] Aukett, et al. Addendum to the IPEMB code of practice for the determination of absorbed dose for x-rays below300 kV generating potential $(0.035 \mathrm{~mm} \mathrm{Al-4} \mathrm{mm}$ Cu HVL). Phys Med Biol 2005;50:2739-48. () 2005 IOP Publishing Ltd.

[27] Lillicrap S, Owen B, Williams J, Williams P. Code of Practice for high-energy photon therapy dosimetry based on the NPL absorbed dose calibration service. Phys Med Biol 1990;35(10):1355-60.
[28] Bassi S, Cummins D, McCavana P. Energy and dose dependence of GafChromic EBT3-V3 film across a wide energy range. Rep Pract Oncol Radiother 2020;25(1): 60-3.

[29] German Standard, Application of X-rays with Tube Voltages between 10 and 100 $\mathrm{kV}$ in Radiotherapy and Soft Tissue Diagnostics, 1988.

[30] CheetahTM material datasheet can be found at this address: Ninjatek Website.

[31] Bassi S, Langan B, Malone C. Dosimetry assessment of patient-specific 3D printable materials for HDR surface brachytherapy. Phys Med 2019;67:166-75.

[32] Hughes I, Hase T. Measurements and their Uncertainties. Oxford: Oxford University Press; 2011.

[33] Low Daniel A, Moran Jean M, Dempsey James F, Dong Lei, Mark Oldham. Dosimetry tools and techniques for IMRT. Med Phys 2011;38(3):1313. https://doi. org/10.1118/1.3514120.

[34] Miften M, Olch A, Mihailidis D, Moran J, Pawlicki T, Molineu A, Li H, Wijesooriya K, Shi J, Xia P, Papanikolaou N, Low D. Tolerance limits and methodologies for IMRT measurement-based verification QA: Recommendations of AAPM Task Group No. 218. Med Phys 2018;45(4):e53-83.

[35] Roué A, Venselaar JLM, Ferreira IH, Bridier A, Van Dam J. Development of a TLD mailed system for remote dosimetry audit for 192Ir HDR and PDR sources. Radiother Oncol 2007;83:86-93. https://doi.org/10.1016/j.radonc.2007.02.011. 